University of Nebraska - Lincoln DigitalCommons@University of Nebraska - Lincoln

\title{
On the Inherent Asymmetric Nature of the Complementary Relationship of Evaporation
}

Jozsef Szilagyi

University of Nebraska-Lincoln, jszilagyi1@unl.edu

Follow this and additional works at: https://digitalcommons.unl.edu/natrespapers

Part of the Natural Resources and Conservation Commons, Natural Resources Management and Policy Commons, and the Other Environmental Sciences Commons

Szilagyi, Jozsef, "On the Inherent Asymmetric Nature of the Complementary Relationship of Evaporation" (2007). Papers in Natural Resources. 956.

https://digitalcommons.unl.edu/natrespapers/956

This Article is brought to you for free and open access by the Natural Resources, School of at DigitalCommons@University of Nebraska - Lincoln. It has been accepted for inclusion in Papers in Natural Resources by an authorized administrator of DigitalCommons@University of Nebraska - Lincoln. 
See discussions, stats, and author profiles for this publication at: https://www.researchgate.net/publication/229048975

\section{On the inherent asymmetric nature of the complementary relationship of evaporation}

Article in Geophysical Research Letters · January 2007

DOI: 10.1029/2006GL028708

CITATIONS

54

1 author:

Joe Szilagyi

Budapest University of Technology and Economics

104 PUBLICATIONS 1,847 CITATIONS

SEE PROFILE

Some of the authors of this publication are also working on these related projects:

Project The Complementary Relationship in Land Surface Evapotranspiration View project
READS

44 


\title{
On the inherent asymmetric nature of the complementary relationship of evaporation
}

\author{
Jozsef Szilagyi ${ }^{1,2}$ \\ Received 8 November 2006; revised 17 December 2006; accepted 19 December 2006; published 24 January 2007.
}

[1] New theoretical considerations indicate that the complementary relationship (CR) of evaporation is inherently asymmetric when the time rate of change between actual and apparent potential evaporations is considered. The theory also estimates the extent of this asymmetry as a function of the surface temperature and predicts that a symmetric CR, independent of the surface temperature, can only be expected when no energy exchange between the source of the apparent potential evaporation process and its surroundings occurs, a rather unrealistic situation. The derived asymmetric CR is employed for operational evaporation estimations. The parameters of the proposed practical evaporation estimation model are from the Priestley-Taylor and Penman equations. Citation: Szilagyi, J. (2007), On the inherent asymmetric nature of the complementary relationship of evaporation, Geophys. Res. Lett., 34, L02405, doi:10.1029/ 2006GL028708.

\section{Introduction}

[2] The complementary relationship (CR) of Bouchet [1963], written in energy flux units, relates actual $(L E)$ and potential evaporation rates $\left(L E_{p}\right)$ to wet environment evaporation $\left(L E_{w}\right)$

$$
L E+L E_{p}=k L E_{w}
$$

where $k$ is a constant of proportionality. Throughout the text the term evaporation includes the transpiration of vegetated surfaces as well. Bouchet did not specify how the different terms should be calculated in (1), thus it took some time before his idea was put into practice by Brutsaert and Stricker [1979] in their Advection Aridity (AA) model.

[3] The definition of potential evaporation is ambiguous due to what constitutes as a 'large area' covered by actively growing vegetation the evapotranspiration of which should be considered under unimpeded access to water [Brutsaert, 2005]. As known, the rate of evaporation of such a moist vegetated area is a function of its size due to the so-called 'oasis effect' of energy transport to the area, the relative importance of which diminishes with the size of the moist surface. In contrast, the application of the apparent potential evaporation [Brutsaert, 2005] is much less ambiguous,

\footnotetext{
${ }^{1}$ Department of Hydraulic and Water Resources Engineering, Budapest University of Technology and Economics, Budapest, Hungary.

${ }^{2}$ Also at School of Natural Resources, University of Nebraska at Lincoln, Lincoln, Nebraska, USA.
}

Copyright 2007 by the American Geophysical Union. 0094-8276/07/2006GL028708\$05.00 since it considers the rate of evaporation under the actual meteorological and surface conditions from a source of water that is small enough in size as to not be able to influence the prevailing conditions of its surroundings. In theory it means that the saturated vapor pressure of the water source is taken at the temperature of the surrounding surface [Brutsaert, 2005]. The latter is rarely known in practice leading one to the application of the Penman equation [Penman, 1948] with data that represent the actual meteorological conditions. As an alternative, the evaporation rate of a pan can be considered as a widely used practical measure of the apparent potential evaporation. In what follows, reference to potential evaporation, $L E_{p}$, will always be considered as the apparent potential evaporation.

[4] In Brutsaert and Stricker's [1979] Advection-Aridity model formulation of the complementary relationship, $L E_{p}$ was calculated with the help of the Penman equation [Penman, 1948]

$$
L E_{p}=\frac{\Delta}{\Delta+\gamma} Q_{n}+\frac{\gamma}{\Delta+\gamma} E_{A}
$$

where $\Delta$ is the slope of the saturation vapor pressure curve at the air temperature, $\gamma$ is the psychometric constant, $Q_{n}$ is the available energy, and $E_{A}$ is the drying power of the air

$$
E_{A}=f(u)\left(e^{*}-e\right)
$$

with $e^{*}$ and $e$ being the saturation (taken at the air temperature) and actual vapor pressure values, respectively, and $f(u)$ is a wind function. The $L E_{w}$ term is specified by the Priestley-Taylor equation [Priestley and Taylor, 1972]

$$
L E_{w}=c \frac{\Delta}{\Delta+\gamma} Q_{n}
$$

where $c$ is the Priestley-Taylor parameter. Employing the so-defined terms of (1), Brutsaert and Stricker [1979] assumed that the $\mathrm{CR}$ is symmetric around the $L E_{w}$ value, i.e.,

$$
L E+L E_{p}=2 L E_{w}
$$

which has become, for its simplicity and practical applicability, widely used.

[5] Recently Kahler and Brutsaert [2006] demonstrated how class A pan evaporation $\left(L E_{p a}\right)$ data, as a practical measure of $L E_{p}$, can be employed to estimate actual evaporation on a daily basis with the help of an inherently symmetric CR. When used with evaporation pan data, 
according to them, the assumed symmetric nature of the CR becomes asymmetric, i.e.,

$$
L E+L E_{p}>2 L E_{w}
$$

is valid instead of an equality. They point out that the asymmetry present in (6) is due to the nature of heat transfer between the pan and its surroundings. As the environment dries, the class A pan receives more energy per unit area than the surrounding land because (a) its side and bottom are exposed, allowing for additional heat transfer in the form of conduction and radiation, and; (b) its small size (and material and thermal properties different from its surroundings) leads to local advection of energy from the surrounding environment. This will mean that the change in the apparent potential evaporation, as the environment dries from an initially wet condition, will be larger than the corresponding change in actual evaporation, i.e.,

$$
L E_{p}-L E_{w}=b\left(L E_{w}-L E\right)
$$

where $b>1$ is another constant of proportionality.

[6] Rearrangement of (7) leads to

$$
L E=\frac{1+b}{b} L E_{w}-\frac{1}{b} L E_{p}
$$

which serves as the operational evaporation estimation method of Kahler and Brutsaert [2006], where for $L E_{p}$ the term $\alpha L E_{p a}$ is inserted, $\alpha$ being a pan coefficient, assumed to be unity. (8) can be considered as a modification of the original AA model specific to pan data.

[7] The major thrust of the present study is to show through theoretical considerations and subsequent data analysis that the asymmetry that arises in (6) is directly and inherently linked to the definition of apparent potential evaporation and not limited only to class A pan measurements. An operational evaporation estimation method, alternative to the original AA model by Brutsaert and Stricker [1979] and its modification by Kahler and Brutsaert [2006], is also derived where the coefficients $b$ and $c$, the latter from the $L E_{w}$ term of (8), come out as well known quantities.

\section{Theoretical Considerations About the Complementary Relationship}

[8] Let's assume that the net energy, $Q_{n}$, available at the surface, is constant through time and that it is fully utilized by the sensible $(H)$ and latent heat $(L E)$ fluxes between the surface and the adjacent air. From an assumed constant $Q_{n}$ term one obtains

$$
\frac{\partial_{t} H}{\partial_{t} L E}=-1
$$

where the operator $\partial$ denotes differentiation with respect to the variable specified in its index (i.e., time here).

[9] For an arbitrarily small area on the surface continuously having the same surface temperature as the drying environment but with unlimited access to water (i.e., representing apparent potential conditions), one can write

$$
\partial_{t} H_{p}=\partial_{t} H
$$

As a working hypothesis let's assume that the ratio of the time rate of change of the evaporation terms belonging to the drying environment $(L E)$ and the small wet area $\left(L E_{p}\right)$ respectively, depends at most on the uniform temperature $\left(T_{S}\right)$ of the surface only (or otherwise it is a constant), i.e.,

$$
\frac{\partial_{t} L E}{\partial_{t} L E_{p}}=f\left(T_{s}\right)
$$

From (9)

$$
\partial_{t} L E=-\partial_{t} H
$$

therefore

$$
\frac{\partial_{t} L E}{\partial_{t} L E_{p}}=\frac{-\partial_{t} H}{\partial_{t} L E_{p}}=f\left(T_{s}\right)
$$

(9) and (10), employing a Dalton-type approach for the sensible and latent heat fluxes, yield

$$
\frac{\partial_{t} H_{p}}{\partial_{t} L E_{p}}=\frac{\partial_{t} H}{\partial_{t} L E_{p}}=\frac{\partial_{t}\left(f_{H} \partial_{z} T\right)}{\partial_{t}\left(f_{L E} \partial_{z} e_{p}\right)}
$$

where $f_{H}=-f(u) c_{p}, f_{L E}=-f(u) 0.622 L / p$ and where it was also considered that near the surface the potential temperature is very close to the actual temperature. Here $f(u)$ is the wind function, $c_{p}$ is the specific heat of air taken at a constant pressure, $L$ is the latent heat of vaporization, $e_{p}$ is the vapor pressure at the completely wet surface of arbitrarily small extent, $p$ is the air pressure and $z$ denotes the vertical direction.

[10] Let's assume now that in (14) the sensible and latent heat fluxes will change predominantly due to the temporally changing vertical gradients of temperature and water vapor as evaporation from the surface, surrounding the arbitrarily small wet patch, decreases due to limited water access and, as a consequence, the warming up of the surface during a dry spell. (This is not to say that the $f_{H}$ and $f_{L E}$ terms would not change with the increasing instability of the air as the environment dries). This way the $f_{H}$ and $f_{L E}$ terms are brought out of the temporal differentiation. Changing the order of differentiation next yields

$$
\frac{\partial_{t} H}{\partial_{t} L E_{p}}=\frac{f_{H} \partial_{t} \partial_{z} T}{f_{L E} \partial_{t} \partial_{z} e_{p}}=\gamma \frac{\partial_{z} \partial_{t} T}{\partial_{z} \partial_{t} e_{p}}=-f\left(T_{s}\right) .
$$

Here the $\gamma=f_{H} / f_{L E}$ term is the psychometric constant. By first rearranging the right-hand-side of (15) (i.e., $\gamma \partial_{z} \partial_{t} T=$ $\left.-f\left(T_{s}\right) \partial_{z} \partial_{t} e_{p}\right)$ and then integrating it with respect to $z$, one obtains

$$
\gamma \partial_{t} T_{s}=-f\left(T_{s}\right) \partial_{t} e_{s}^{*}+F(t)
$$

where $F(t)$ is the integral constant that can only depend on time or be a simple constant. The $e_{p}$ term now becomes $e_{s}^{*}$, 
the saturation vapor pressure at the surface temperature, $T_{S}$, since the vertical gradient terms dropped out. The dependence of the $\gamma$ on $z$ was neglected. $F(t)$ cannot be a function of time, since a shift in the time reference should not change the results, so it can only be a constant. But that cannot be different from zero since the time rate of change for $T_{s}$ is zero only when the same for $e_{s}^{*}$ is zero and vice versa. Note that under a constant $Q_{n}$ condition the vertical gradients of the temperature and vapor pressure terms cannot change independently either, by virtue of (9) and (15). Also, the $\partial_{z} \partial_{t} T / \partial_{z} \partial_{t} e_{p}$ term in (15) can only be replaced by the $\partial_{t} T_{s} / \partial_{t} e_{s}^{*}$ term from (16) (obtained with $F(t)=0$ and both sides divided by $\left.\partial_{t} e_{s}^{*}\right)$ if there is a change in the surface temperature of the wet patch to avoid an indeterminable expression in the latter.

[11] By combining (15) and (16) with $F(t)=0$ leads to

$$
\frac{\partial_{t} H}{\partial_{t} L E_{p}}=\gamma \frac{\partial_{t} T_{s}}{\partial_{t} e_{s}^{*}}=-f\left(T_{s}\right)
$$

(17) can be written as

$$
\frac{\partial_{t} H}{\partial_{t} L E_{p}}=\frac{\gamma}{\Delta_{s}}
$$

where $\Delta_{S}$ is the slope of the saturation vapor pressure curve at $T_{s}$, the surface temperature.

[12] From (12) and (18) one obtains

$$
\frac{\partial_{t} L E}{\partial_{t} L E_{p}}=\frac{\partial L E}{\partial L E_{p}}=-\frac{\gamma}{\Delta_{s}}
$$

which corroborates the working hypothesis that the ratio of the time rate of change of the actual and apparent potential evaporation terms is indeed a function of the surface temperature, and not a constant, as a symmetric CR would suggest.

[13] From the above it follows that the CR is symmetric only, independent of the surface temperature, when there exists no energy transfer between the source of the apparent potential evaporation and its surroundings. When this latter happens, the energy available for the apparent potential evaporation process remains constant, therefore (9) is valid

$$
\frac{\partial_{t} H_{p}}{\partial_{t} L E_{p}}=-1
$$

Combining it with (17) yields

$$
\frac{\partial_{t} H_{p}}{\partial_{t} L E_{p}}=\gamma \frac{\partial_{t} T_{s p}}{\partial_{t} e_{S}^{*}}=-1
$$

which can never happen as long as the wet surface temperature, $T_{s p}$, changes in time. When, however, $T_{s p}$ is constant, (21), in order to avoid an indeterminable $0 / 0$ expression, must be written with the vertical gradients taken at the wet surface, i.e.,

$$
-1=\gamma \frac{\partial_{z} \partial_{t} T_{p}}{\partial_{z} \partial_{t} e_{p}}
$$

which is guaranteed to be negative since the numerator is always positive, and the denominator is the opposite, as the environment dries up. From (9) and (20) the CR now becomes symmetric

$$
\frac{\partial L E}{\partial L E_{p}}=-1
$$

with the customary $\partial H=\partial L E_{p}$ assumption [e.g., Hobbins et al., 2001], independent of the surface temperature.

[14] As a summary the theory predicts that the CR should generally be asymmetric in nature when the time rate of change between actual and apparent potential evaporation is considered. It specifies the degree of asymmetry as a function of the surface temperature yielding an equal but opposite change in the two evaporation terms provided the surface temperature is about $6^{\circ} \mathrm{C}$, i.e., when $\gamma=\Delta$ at around $1000 \mathrm{hPa}$. The theory further predicts that the CR can be symmetric, independently of the surface temperature, only when there is no energy exchange between the source of apparent potential evaporation and the drying environment [Szilagyi, 2001], which is a very unrealistic condition.

[15] The explanation of the predicted asymmetry of the $\mathrm{CR}$ lies in the energy supply the drying environment, characterized by increasing surface and air temperatures, provides toward the source of the apparent potential evaporation to elevate its surface temperature, which would otherwise stay constant as was shown above. This way the apparent potential evaporation process locally consumes more energy than available overall from an assumed constant $Q_{n}$ term. This energy transfer is universally present in reality mainly as (a) advection of warmer air over the small wet area, and; (b) heat conduction in the ground from the surrounding warmer and dryer soil.

[16] The present theory considers two extremes, on one hand, when the heat conduction is perfect and the wet area achieves the same surface temperature as the drying environment, and on the other hand, when there is no energy transfer at all between the two areas having different wetness and therefore different surface temperatures. The application of the Penman equation is a proxy for the perfect heat conduction case as it employs drying environment characteristics. In reality, practical measures of apparent potential evaporation, such as the application of an evaporation pan or an evaporimeter found at standard meteorological stations, may express a degree of asymmetry between (and perhaps beyond for evaporation pans due to differences in surface properties between the pan as well as its water and the surrounding vegetated land) the two theoretical extremes considered.

\section{Formulation and Testing of the Practical Operational Evaporation Estimation Model and Discussion}

[17] The above results can be utilized in formulating an operational evaporation model. (19) written in finite differences between the actual and wet environment values, i.e., when $L E=L E_{p}=L E_{w}$, and after rearrangement yields

$$
L E=\left(1+\frac{\gamma}{\Delta^{*}}\right) L E_{w}-\frac{\gamma L E_{p}}{\Delta^{*}}=L E_{w}+\frac{\gamma}{\Delta^{*}}\left(L E_{w}-L E_{p}\right)
$$


(a)

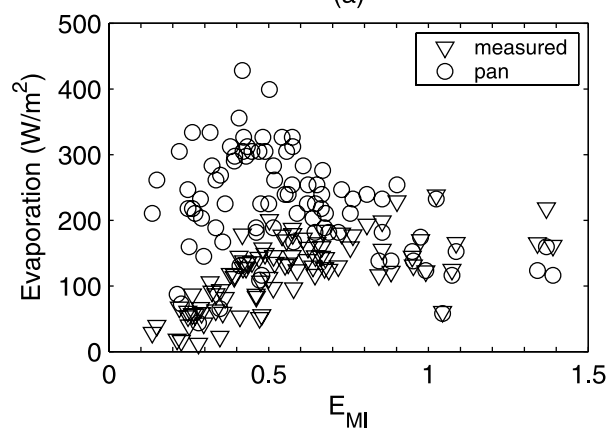

$\mathrm{MI}$
$(\mathrm{c})$

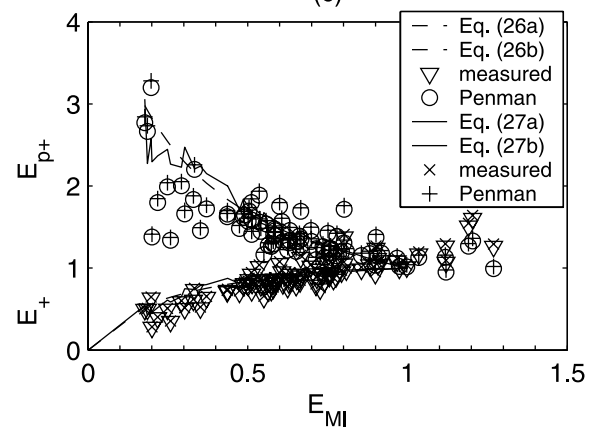

(b)

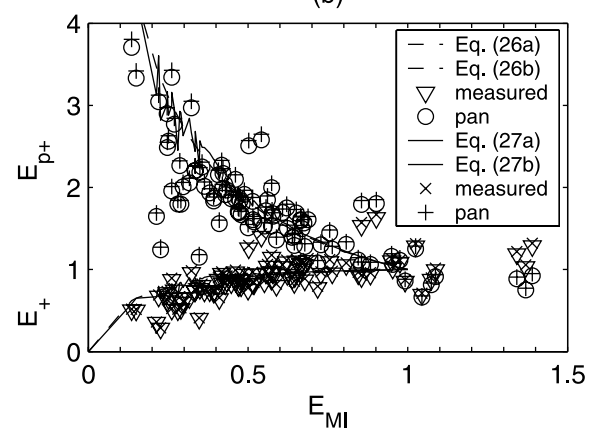

(d)

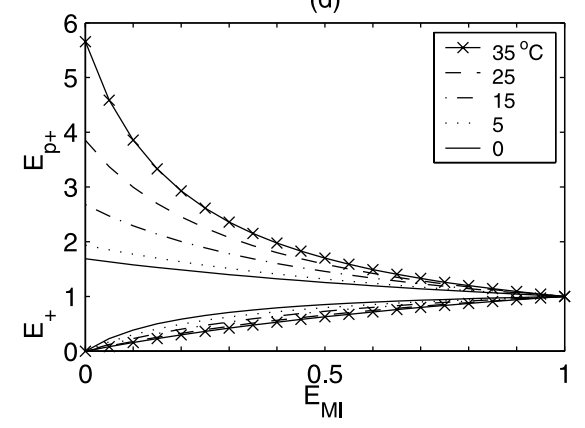

Figure 1. (a) Daily actual $(L E)$ and pan evaporation $\left(L E_{p a n}\right)$ data for 1987 and 1989 near Manhattan, $K S . E_{M I}=L E / L E_{p a n}$. (b) $L E_{w}$-normalized values of actual $\left(E_{+}\right)$and pan evaporation $\left(E_{p+}\right)$ with $c=1.18$ (hollow symbols) and $c=1.15$. The curves represent (26) and (27) with $b=9.45$ and $\varepsilon=4.71$, respectively, $E_{M I}=L E / L E_{p a n}$. (c) $L E_{w}$-normalized values of actual $\left(E_{+}\right)$and Penman-equation (PM) evaporation $\left(E_{p+}\right)$ with $c=1.2$ (hollow symbols) and $c=1.17$. The curves represent (26) and (27) with $b=4.21$ and $\varepsilon=2.24$, respectively, $E_{M I}=L E / L E_{P M}$. (d) Dimensionless graph of the complementary relationship as predicted by (27) with $\varepsilon=1$.

where the slope of the saturation vapor pressure curve is taken with an effective value, $\Delta^{*}$. This is necessary, because $\Delta_{s}$ is a function of the changing surface temperature, the value of which is not known even under reference wet environment conditions. Since in practice not only the reference wet environment surface temperature is unknown but so is the actual surface temperature, the effective temperature at which $\Delta^{*}$ is calculated must somehow be related to the only known temperature value, which is the actual air temperature. The simplest approach is through a constant, $\varepsilon$, i.e., $\Delta^{*}=\varepsilon \Delta$, where $\Delta$ is the slope of the saturated vapor pressure curve taken at the actual air temperature. This way (24) becomes

$$
L E=\left(1+\frac{\gamma}{\varepsilon \Delta}\right) L E_{w}-\frac{\gamma L E_{p}}{\varepsilon \Delta}=L E_{w}+\frac{\gamma}{\varepsilon \Delta}\left(L E_{w}-L E_{p}\right)
$$

which is the practical evaporation estimation model. (25) is similar to (8) except $b=\varepsilon \Delta / \gamma$ now.

[18] For the present analysis, data from the First International (Satellite-Land Surface Climatology) Field Experiment (FIFE) were employed. This includes two Bowen-ratio flux measurement stations for the periods May 26-October 17 in 1987 (station \#40) and July 21-August 14 in 1989 (station \#944); an eddy-correlation station with the longest available continuous record for 1987 (station \#26) as well as a class A pan at the Tuttle Creek dam near Manhattan, Kansas. For a general description of the measurement site and experimental settings, please, refer to Kahler and Brutsaert [2006]. Note that (a) in 1987 the revised data set of FIFE was employed; (b) the surroundings of the pan are far from ideal. The pan not only sits $300 \mathrm{~m}$ directly south of the dam as reported by Kahler and Brutsaert [2006], but it is also a few hundred meters from a sizeable lake (River Pond State Park) to the south. This fact certainly contributes to some uncertainties in the ensuing data analysis, which involves this pan in relation to the CR. Consequently, choosing the days with certain predominant wind directions [Kahler and Brutsaert, 2006] has been abandoned and all available records for the time period were retained instead. The flux measurement stations \#40 and \#944, distributed on the FIFE CDs, did not include air temperature data, so for that the meteorological station at Kansas State University in Manhattan was used in the analysis. The data required by the Penman equation for calculating the drying power of the air were from the NCDC (for 1989) and NOAA (for 1987) surface measurement stations in Manhattan, also included in the FIFE CDs.

[19] Daily evaporation flux was calculated as the mean of the two flux stations' daily values in 1987 when both sources of data were available. Figure 1a displays these daily actual and pan evaporation values as a function of a soil moisture index, $E_{M I}=L E / L E_{p a}$. The data scatter in Figure 1a can be reduced (Figure $1 \mathrm{~b}$ ) by normalizing the $L E$ and $L E_{p a}$ values with $L E_{w}$ of (4). From (8) the normalization yields

$$
E_{+}=\frac{(1+b) E_{M I}}{1+b E_{M I}}
$$




$$
E_{p+}=\frac{1+b}{1+b E_{M I}}
$$

Performing a nested trial-and-error optimization routine for both coefficients $c$ and $b$ in (8), and accepting an updated value of the parameters only when the reduction in the ensuing sum of squared-error (SSE) is larger than $1 \%$ of the previous value, Figure $1 \mathrm{~b}$ results with $c=1.18$ and $b=9.45$. Here an interesting property of the dimensionless graph of Figure 1b ought to be mentioned. Namely, the curve optimized via (8) is not, and will not be, the best-fit curve of the dimensionless graph. A short proof is given in Appendix A. As mentioned by Kahler and Brutsaert [2006], a moisture index $\left(E_{M I}\right)$ that is derived independently from actual evaporation to be estimated would be preferable to the present formulation, where the index itself contains the desired evaporation value. Such a choice for $E_{M I}$ could be the ratio of $L E_{w}$ and $L E_{p}$. Application of this new moisture index, however, necessarily results in a compressed range of $E_{M I}$ values (i.e., $0.5-1$ for a $b=1$ choice and somewhat larger for $b>1$ ), and while it circumvents the problem of obtaining differing optimal parameter values of $c$ and $b$ depending on whether the optimization is based on dimensionless or original data, mentioned above, it leads to such a cloud of $E_{+}$in the dimensionless graph that a clear complementarity between $L E_{p}$ and $L E$ becomes difficult to detect. As a consequence, the results of the ensuing analysis will be demonstrated with Kahler and Brutsaert's [2006] original moisture index. Note, however, that both indices result in the same estimates of $c$ and $b$, as long as the optimization is based on (8).

[20] Figure 1b also displays the evaporation estimates based on an optimization of (25), approximating $L E_{w}$ with (4) and $L E_{p}$ with the pan readings, along with the measured evaporation flux data. Again, the normalization of (25) by $L E_{w}$ yields

$$
\begin{aligned}
& E_{+}=\frac{(1+\varepsilon \Delta / \gamma) E_{M I}}{1+\varepsilon \Delta E_{M I} / \gamma} \\
& E_{p+}=\frac{1+\varepsilon \Delta / \gamma}{1+\varepsilon \Delta E_{M I} / \gamma}
\end{aligned}
$$

The values of $\varepsilon$ and $c$ became 4.71 and 1.15 , respectively. The fit between observed and predicted $E_{+}$values remained the same, i.e., the root-mean-square-error, $\mathrm{RMSE}=0.18$.

[21] For further testing (Figure 1c), the Penman [1948] equation with its original wind function [Brutsaert, 2005] was used in place of the evaporation pan measurements to estimate $L E_{p}$ [Crago and Crowley, 2005]. The optimized value of $b$ became 4.21 while the optimized value of $c$ changed only slightly to 1.2 . The explained variance by the model $\left(\mathrm{R}^{2}\right)$ remained the same as with the pan values, 0.75 . When the same optimization was next performed with (25), the optimized value of $\varepsilon$ and $c$ became 2.24 and 1.17, respectively. The $\mathrm{R}^{2}$ value did not change, it remained 0.75 , as with the pan data. The fit between observed and theoretical values improved in Figure 1c compared with Figure $1 \mathrm{~b}$ with an $\mathrm{RMSE}=0.12$ now for both methods.

[22] There remained the formulation of a practical operational evaporation model that is based on the predicted asymmetric property of the $\mathrm{CR}$ and does not contain any parameters to be tuned. Since the optimized value of the original model's $b$ in (8) (as shown by Kahler and Brutsaert [2006]) or the revised model's $\varepsilon$ parameter (being close to unity) in (25), may change from place to place or from season to season (due to changes, e.g., in vegetation), the practical operational model can have a predefined value of $\varepsilon=1$. Choosing of a suitable $c$ value was achieved by optimizing (25) with an $\varepsilon$ value of unity employing both pan evaporation and Penman equation values. With the pan data, the optimized value of $c$ became $1.31\left(\mathrm{R}^{2}=0.62\right)$, while with the Penman equation values it became $1.24\left(\mathrm{R}^{2}=\right.$ $0.72)$. These two values bracket tightly the mean value of 1.26 of the same coefficient reported by Priestley and Taylor [1972]. Consequently, the practical operational model can have a $c$ value of 1.26. The practical evaporation model better performs (as expected) with the Penman $\left(\mathrm{R}^{2}=\right.$ $0.72)$ than with the pan values $\left(R^{2}=0.62\right)$ and its performance is almost the same as that of its fully optimized version.

[23] This same practical evaporation model $(\varepsilon=1$ and $c=1.26$ ) has been first applied by Crago and Crowley [2005] in a large comparative study involving six other CRbased evaporation estimation models and performed by far the best. Their best performing evaporation estimation model was criticized by Lhomme and Guilioni [2006] for being built on faulty arguments by Granger [1989]. Interestingly, the present theoretical considerations, completely different from Granger's [1989], have lead to the same practical model as was first proposed by him and recently tested by Crago and Crowley [2005].

[24] Finally, Figure 1d depicts the CR in a dimensionless form as expressed by (27) with $\varepsilon=1$. The CR is symmetric only when the surface temperature is about $6^{\circ} \mathrm{C}$ (and air pressure is $1000 \mathrm{hPa}$ ), and asymmetric otherwise. With (2) and (4) for the $L E_{p}$ and $L E_{w}$ terms the CR thus becomes

$$
\begin{array}{ll}
L E+L E_{p}<2 L E_{w}, & T_{s}<6^{\circ} C \\
L E+L E_{p} \approx 2 L E_{w}, & T_{s} \approx 6^{\circ} C \\
L E+L E_{p}>2 L E_{w}, & T_{s}>6^{\circ} C
\end{array}
$$

Note that the temperature at which the CR becomes symmetric changes with elevation due to the pressure dependence of the psychometric constant.

[25] The above illustrated asymmetric nature of the CR not only helps interpret the results of Kahler and Brutsaert [2006] in a more general way, but it may also explain why (a) Crago and Crowley [2005] obtained the best evaporation estimates with the (25)-modified AA model (with $\varepsilon=1$ ) out of six CR-based evaporation estimation methods, and; (b) Szilagyi et al. [2001] observed a pan evaporation decrease of about $6 \%$ over the conterminous US in the period of 1948-1996, accompanied only by an about $3 \%$ increase in actual evaporation. It very likely also explains why Hobbins et al. [2001] had to optimize the original AA model (built on a symmetric CR) of Brutsaert and Stricker [1979] locally to match the predictive power of another CR-based model by Morton [1983]. Note that in the Morton model the $L E_{w}$ term is purposefully defined (differently from Priestley and Taylor [1972]) in a way that compels his formulation of the CR to be symmetric, as was pointed out 
by Granger [1989]. Furthermore, the asymmetric nature of the $\mathrm{CR}$ discussed here obviates the need of correcting the pan measurements of Ramirez et al. [2005] to bring their data in accordance with an expected symmetric CR. Finally, it also provides an explanation of the observed asymmetric relationship between actual and apparent potential evaporation that was obtained by Pettijohn and Salvucci [2006] with their FIFE data analyzed, different from the one reported here.

\section{Appendix A}

[26] The objective function of the parameter optimization must have a minimum with the optimized values of the parameters, i.e.,

$$
\sum\left\{L E-\left[\left(\frac{1+b}{b}\right) L E_{w}-\frac{L E_{p}}{b}\right]\right\}^{2}=\min
$$

where we omitted the indices for simplicity. From (26a) the so-derived parameter values will lead to a best-fit curve in Figures $1 \mathrm{~b}$ and $1 \mathrm{c}$ provided

$$
\sum\left\{\frac{L E}{L E_{w}}-\frac{(1+b) L E / L E_{p}}{1+b L E / L E_{p}}\right\}^{2}=\min
$$

also. This last condition holds true only, if the estimate of $L E$ in (A2) is a (yet unspecified) function of that of (A1). But this is clearly not the case, since the estimate of $L E$ in (A2) contains $L E$ itself, which is not of a strictly functional relationship (due to, e.g., measurement error) with either $L E_{w}$ or $L E_{p}$. This concludes the proof.

[27] Acknowledgments. The author is grateful to Rich Crago and to a second anonymous reviewer whose comments led to important revisions.

\section{References}

Bouchet, R. J. (1963), Evapotranspiration reelle, evapotranspiration potentielle, et production agricole, Ann. Agron., 14, 743-824.

Brutsaert, W. (2005), Hydrology: An Introduction, 605 pp., Univ. of Cambridge Press, New York.

Brutsaert, W., and H. Stricker (1979), An advection-aridity approach to estimate actual regional evapotranspiration, Water Resour. Res., 15, $443-449$

Crago, R., and R. Crowley (2005), Complementary relationships for nearinstantaneous evaporation, J. Hydrol., 300, 199-211.

Granger, R. J. (1989), A complementary relationship approach for evaporation from nonsaturated surfaces, J. Hydrol., 111, 31-38.

Hobbins, M. T., J. A. Ramírez, and T. C. Brown (2001), The complementary relationship in estimation of regional evapotranspiration: An enhanced advection-aridity model, Water Resour. Res., 37, 1389-1403.

Kahler, D. M., and W. Brutsaert (2006), Complementary relationship between daily evaporation in the environment and pan evaporation, Water Resour. Res., 42, W05413, doi:10.1029/2005WR004541.

Lhomme, J. P., and L. Guilioni (2006), Comments on some articles about the complementary relationship, J. Hydrol., 323, 1-3.

Morton, F. I. (1983), Operational estimates of areal evapotranspiration and their significance to the science and practice of hydrology, J. Hydrol., 66, $1-76$.

Penman, H. L. (1948), Natural evaporation from open water, bare soil, and grass, Proc. R. Soc. London, Ser. A, 193, 120-146.

Pettijohn, J. C., and G. D. Salvucci (2006), Impact of an unstressed canopy conductance on the Bouchet-Morton complementary relationship, Water Resour. Res., 42, W09418, doi:10.1029/2005WR004385.

Priestley, C. H. B., and R. J. Taylor (1972), On the assessment of surface heat flux and evaporation using large-scale parameters, Mon. Weather Rev., 100, 81-92.

Ramírez, J. A., M. T. Hobbins, and T. C. Brown (2005), Observational evidence of the complementary relationship in regional evaporation lends strong support for Bouchet's hypothesis, Geophys. Res. Lett., 32, L15401, doi:10.1029/2005GL023549.

Szilagyi, J. (2001), On Bouchet's complementary hypothesis, J. Hydrol., $246,155-158$.

Szilagyi, J., G. G. Katul, and M. B. Parlange (2001), Evapotranspiration intensifies over the conterminous United States, J. Water Resour. Plann. Manage., 127(6), 354-362.

J. Szilagyi, School of Natural Resources, University of Nebraska at Lincoln, Lincoln, NE 68588, USA. (jszilagyi1@unl.edu) 